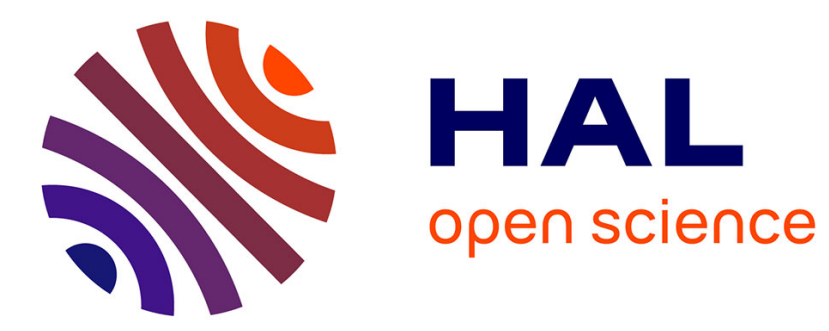

\title{
Anisotropic unstable ion distribution functions downstream of the solar wind termination shock
}

H.-J. Fahr, M. Siewert

\section{To cite this version:}

H.-J. Fahr, M. Siewert. Anisotropic unstable ion distribution functions downstream of the solar wind termination shock. Astrophysics and Space Sciences Transactions, 2007, 3 (1), pp.21-27. hal00297486

\section{HAL Id: hal-00297486 \\ https://hal.science/hal-00297486}

Submitted on 19 Sep 2007

HAL is a multi-disciplinary open access archive for the deposit and dissemination of scientific research documents, whether they are published or not. The documents may come from teaching and research institutions in France or abroad, or from public or private research centers.
L'archive ouverte pluridisciplinaire $\mathbf{H A L}$, est destinée au dépôt et à la diffusion de documents scientifiques de niveau recherche, publiés ou non, émanant des établissements d'enseignement et de recherche français ou étrangers, des laboratoires publics ou privés. 


\title{
Anisotropic unstable ion distribution functions downstream of the solar wind termination shock
}

\author{
H.-J. Fahr and M. Siewert \\ Argelander Institute for Astronomy, University of Bonn, Auf dem Hügel 71, 53121 Bonn, Germany
}

Received: 18 June 2007 - Revised: 8 August 2007 - Accepted: 13 August 2007 - Published: 19 September 2007

\begin{abstract}
In this paper we demonstrate that solar wind ions, passing over the quasiperpendicular portion of the solar wind MHD termination shock, unavoidably develop strongly pronounced pitchangle anisotropies. In order to prove that, we solve the Boltzmann - Vlasov equation for the ions, kinetically describing the ion passage over the MHD structure of the shock. With the solution of the anisotropic downstream ion distribution function we may also calculate higher order velocity moments of this distribution enabling us to calculate anisotropic downstream ion pressures. From these latter results we derive the conclusion that in most likely cases the downstream ion distribution will be mirror-mode unstable and with its free thermal energy will effectively drive magnetosonic turbulences. We estimate the energy that is pumped into this turbulence until marginal stability is achieved. In this newly established intermediate quasi-equilibrium state, as we can show, one can find 35 to 50 percent of the original energy sitting in the thermal mode perpendicular to the magnetic field in the form of magnetosonic turbulences, perhaps already identified by Voyager- 1 as downstream trains of magnetic holes and humps. We discuss several consequences of this new quasi-equilibrium MHD plasma state downsstream of the shock.
\end{abstract}

\section{Introduction}

Usually, the solar wind termination shock is described as an abrupt change of lowest order MHD moments of the plasma distribution function. This change is usually characterized by Rankine-Hugoniot shock relations only taking care of the conservation of the most important fluxes like mass-, momentum-, and energy- fluxes (see e.g. Hudson, 1970; Gombosi, 1998; Erkaev et al., 2000; Diver, 2001). In these relations the corresponding downstream plasma quan-

Correspondence to: H.-J. Fahr (hfahr@astro.uni-bonn.de)

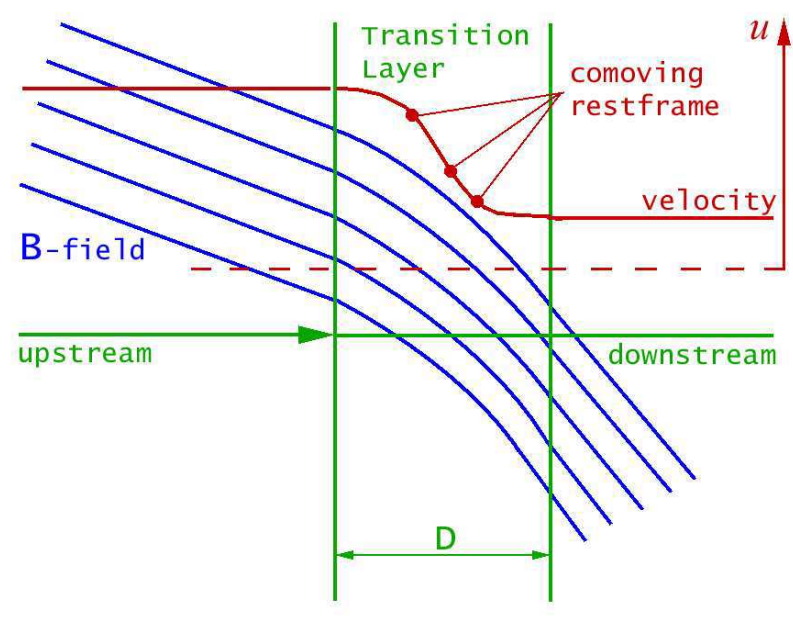

Fig. 1. A simple sketch of how the transition of an ion plasma flow from the upstream side to the downstream side of a MHD shock may look like.

tities are given as functions of the upstream quantities, disregarding the microphysics that arranges for these changes and the structure of the transition region over which these changes have to take place. In general the plasma is described as a mono-fluid, with electrons simply guaranteeing the quasineutrality and with ideally low electrical resistivity guaranteeing frozen-in magnetic fields. In Fig. 1 we give an illustration of the transition from upstream to downstream MHD quantities which should occur under such conditions.

The phenomenological appearance of MHD shocks can, however, look very much different and much more complicate than presented in the view sketched in this figure. This was already demonstrated in test particle studies by Lee et al. (1986) or Quest (1986) and has also been recognized by more sophisticated MHD shock simulations (see Lembege et al.,

Published by Copernicus Publications on behalf of the Arbeitsgemeinschaft Extraterrestrische Forschung e.V. 


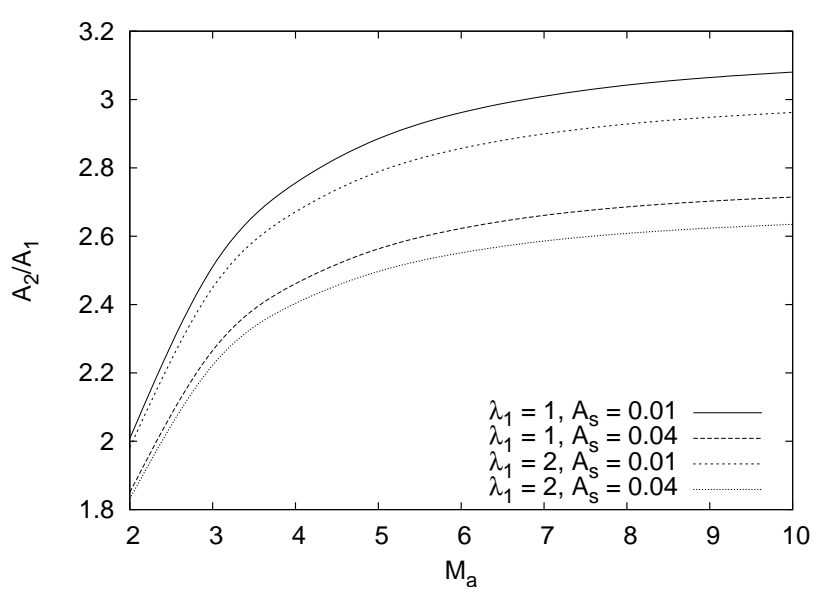

Fig. 2. The downstream pressure anisotropies $A_{2}$ for an isotropic $\left(A_{1}=1\right)$ and anisotropic $\left(A_{1}=2\right)$ upstream plasma as a function of the compression ratio $\sigma$, the Alfvenic Mach number $M_{a 1}$, and the sonic Mach number index $A_{S}=M_{S}^{-2}$.

2004; Krasnoselskikh et al., 2002; Scholer et al., 2003; Scholer and Matsukiyo, 2004; Scholer, 2004) and recent Cluster and Voyager measurements (Lobzin et al., 2007; Kiraly, 2005; Zank et al., 2004). All these studies clearly point towards the idea that, instead of only the lowest MHD moments, full kinetic descriptions of the plasma passing over the shock should be aimed at, including a description of electrons as a separate plasma population (see Siewert and Fahr, 2007b).

\section{A kinetic approach to the shock}

The kinetic properties of the distribution function of ions passing over the quasiperpendicular portion of the solar wind termination shock can be adequately described by a Boltzmann-Vlasov equation in the form developed by Fahr and Siewert (2006). One can solve this Boltzmann-Vlasov equation, written in the velocity coordinates of the comoving plasma bulk frame and describing the ion distribution as function of the line element $s$ measured along the shock normal, by integrating the differential equation (for details see Siewert and Fahr, 2007a)

$$
\frac{d}{d s} \bar{f}\left(w_{\|}, w_{\perp}, s\right)=\left\{\left(\frac{w_{\perp}}{2 B} \frac{d B}{d s}\right) \frac{d}{d w_{\perp}}\right\} \bar{f}\left(w_{\|}, w_{\perp}, s\right)
$$

Here $\bar{f}\left(w_{\|}, w_{\perp}, s\right)$ is the ion distribution function in the plasma bulk frame written as function of the space coordinate $s$ and of the velocity coordinates $w_{\|}, w_{\perp}$ parallel and perpendicular to the local magnetic field $B$, respectively. The above differential equation can be solved by integration, starting from the upstream boundary distribution function $\bar{f}\left(w_{\|}, w_{\perp}, s=-s_{\infty}\right)=\bar{f}_{1}\left(w_{\|}, w_{\perp}\right)$ and delivering the downstream distribution function $\bar{f}\left(w_{\|}, w_{\perp}, s=+s_{\infty}\right)=\bar{f}_{2}\left(w_{\|}, w_{\perp}\right)$.

From this distribution function one of the most interesting higher velocity moments, namely the resulting downstream pressure anisotropy, can be obtained as ratio of the following two moments of $\bar{f}_{2}\left(w_{\|}, w_{\perp}\right)$

$A_{2}=\frac{P_{\perp 2}}{P_{\| 2}}=\frac{\int d^{3} w w_{\perp}^{2} \bar{f}_{2}\left(w_{\|}, w_{\perp}\right)}{\int d^{3} w w_{\|}^{2} \bar{f}_{2}\left(w_{\|}, w_{\perp}\right)}$.

After solving the Boltzmann equation (Eq. (1)) this expression evaluates to (see Fahr and Siewert, 2006; Siewert and Fahr, 2007a)

$A_{2}=\frac{\sigma^{2} P_{\perp 1}}{\sigma P_{\| 1}}=\sigma A_{1}$,

where $\sigma=n_{2} / n_{1}$ denotes the shock compression ratio. In Fig. 2, we demonstrate how the resulting downstream ion pressure anisotropy $A_{2}$ behaves as function of the upstream Alfvenic Machnumber $M_{a 1}$, or, respectively, the compression ratio $\sigma$ of the termination shock (TS). From this figure one may see that, starting from an isotropic upstream distribution function (i.e. $A_{1}=1$ ) one finally ends up at the downstream side of the TS with a highly anisotropic ion distribution function of an anisotropy of $A_{2} \geq 2.6$, if the shock is characterized by an upstream Machnumber $M_{a 1} \geq 6$.

The point generally not taken into account is that after passage over the TS the plasma ions do show a pronounced pitchangle anisotropic distribution function. The resulting downstream distribution function $\bar{f}_{2}\left(w_{\|}, w_{\perp}\right)$, just after passage over the TS, may even be unstable with respect to the so-called mirror mode instability which emerges when (see Baumjohann and Treumann, 1996)

$\frac{\beta_{\perp}}{\beta_{\|}} \geq \frac{1+\beta_{\perp}}{\beta_{\perp}}$

is fulfilled, where the usual definitions of the plasma betavalues have been used, i.e.

$\beta_{\perp, \|}=\frac{P_{\perp, \|}}{\left(B^{2} / 8 \pi\right)}$.

The mirror mode instability drives low frequency $\left(\omega \ll \Omega_{g}=e B / m_{p} c\right)$ magneto-acoustic or magneto-sonic waves with wavevectors $|\boldsymbol{k}| \simeq k_{\perp}$. The energy $\epsilon_{\text {turb }}$ per unit volume of the integrated wave power of this magnetosonic turbulence, which in total can be transfered from free thermal ion energies into these turbulences downstream of the shock, can be estimated by

$\epsilon_{\text {turb }} \simeq\left(P_{2 \perp}-\tilde{P}_{2 \perp}\right)$,

where $P_{2 \perp}$ and $\tilde{P}_{2 \perp}$ are the two relevant downstream pressures, the former resulting immediately after Liouvillean shock passage and the latter after a mirror-mode driven relaxation to the marginally mirror-mode stable pressure (i.e. 
$\left.\tilde{A}_{2} \leq \beta_{2 \perp}+\left(1 / \beta_{2 \perp}\right)\right)$ has happened, respectively. The latter quantity can be calculated under the assumption that during the relaxation process the pressure $P_{2 \|}$ stays essentially unchanged, which then amounts to

$\frac{\tilde{\beta}_{2 \perp}}{\beta_{2 \|}}=\frac{1+\tilde{\beta}_{2 \perp}}{\tilde{\beta}_{2 \perp}}$.

This yields for the marginally stable pressure $\tilde{P}_{2 \perp}$ the following result:

$\tilde{P}_{2 \perp}=\frac{P_{2 \|}}{2}+\sqrt{\frac{P_{2 \|}^{2}}{4}+\frac{B_{2}^{2} P_{2 \|}}{8 \pi}}$.

From the above we then obtain

$\epsilon_{\text {turb }} \simeq\left(P_{2 \perp}-\frac{P_{2 \|}}{2}-\sqrt{\frac{P_{2 \|}^{2}}{4}+\frac{B_{2}^{2} P_{2 \|}}{8 \pi}}\right)$,

which furthermore leads to

$\epsilon_{\text {turb }} \simeq P_{2 \perp}\left(1-\frac{1}{A_{2}}\left(\frac{1}{2}+\sqrt{\frac{1}{4}+\frac{1}{\beta_{2 \|}}}\right)\right)$.

For weakly magnetized plasmas, i.e. high $\beta$-values, and anisotropies of $A_{2} \simeq 2.6$, we then obtain

$\epsilon_{\text {turb }} \simeq P_{2 \perp}\left(1-\frac{1}{A_{2}}\right)=P_{2 \perp}\left(1-\frac{3}{5.2}\right)=0.62 P_{2 \perp}$.

Results for more general cases are shown in Fig. 3. In configurations descibed by Eq. (1), this points to the unexpected fact that about 60 percent or more of the shockentropized upstream kinetic ion energy may be converted into downstream magneto-acoustic turbulences, even in the first step. These increased downstream turbulence levels may then have a strong impact on co-existing ion spectra via nonlinear wave-particle interactions, which in turn may drive the wave-plasma system towards a new quasi-equilibrium state further downstream of the shock, perhaps along the way that has been described by Fisk and Gloeckler (2006), who find arguments why the isotropic ion distribution functions should tend towards a power law with a specific power index of $\gamma_{w}=-5$, when the system allows for an equilibrium in which per time unit as much turbulent energy accelerates ions as work is done by the ions against the turbulent wave pressures.

Assuming that such a state is characteristic for the upstream ion distribution which is convected over the TS, one can then start from an expression for the angle-averaged distribution function like

$f_{1}(w)=\Phi_{1}\left(w / w_{0}\right)^{-5}$,

with $\Phi_{1}$ being a constant taking care of the normalization of the function, and $w_{0}$ being a reference value of the velocity

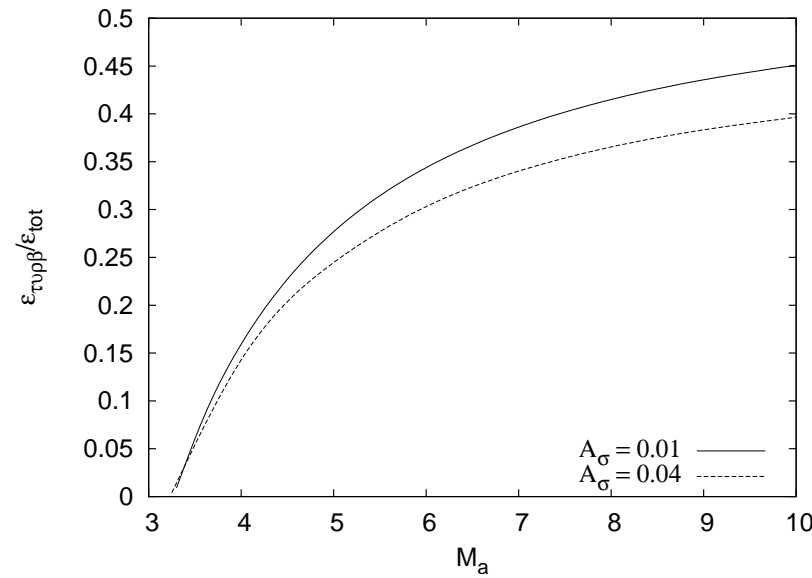

Fig. 3. $\epsilon_{\text {turb }} / \epsilon_{\text {tot }}$ as a function of $M_{a 1}$ and using consistent values for the plasma- $\beta$.

magnitude $w$. Now, we need to apply our anisotropic model, using

$\begin{aligned} w_{\|} & =w \cos \alpha \\ w_{\perp} & =w \sin \alpha,\end{aligned}$

where $\alpha$ is the pitch angle. However, since Fisk and Gloeckler (2006) are using an isotropic model, while we are using an explicitly anisotropic one, making the comparision far from trivial. However, as an approximation, we may select the representations

$f_{1}=\Phi_{1} \cdot\left(\frac{w_{\perp}}{w_{0} \sin \alpha}\right)^{-5}$

and

$f_{1}=\Phi_{1} \cdot\left(\frac{w_{\|}}{w_{0} \cos \alpha}\right)^{-5}$.

These approximations then lead to (Siewert and Fahr, 2007a)

$f_{2}=\Phi_{1} \cdot\left(\frac{w_{\perp}}{w_{0} \sqrt{\sigma} \sin \alpha}\right)^{-5}=\Phi_{1} \cdot\left(\frac{w}{w_{0} \sqrt{\sigma}}\right)^{-5}$

and

$f_{2}=\Phi_{1} \cdot\left(\frac{w_{\|}}{w_{0} \cos \alpha}\right)^{-5}=f_{1}$

The formalism leading to these results is based on a separation ansatz, which is not possible for a power law in an isotropic model; however, very recently we were able to prove that this result is likewise valid when this is not fulfilled (Siewert and Fahr, 2007b). Even without averaging out the anisotropy, which would be required to compare our results to those indicated by Fisk and Gloeckler (2006), it is obvious that these two differing results may only be considered as two extreme boundary configurations, and the true 
result should be somewhere between them, which seemingly does not allow us to compare both models easily.

However, this task is greatly simplified by noting that our results are very similar to the earlier results obtained by Fahr and Lay (2000), where a model assuming global isotropy on both sides of the shock was analyzed. There, it was found that

$f_{2}(w)=\frac{1}{\sqrt{\sigma}} f_{1}\left(\frac{w}{\sigma}\right)$

which was based on arguments comparable to the ones used in Siewert and Fahr (2007b), leading to

$f_{2}(w)=\frac{\Phi_{1}}{\sqrt{\sigma}} \cdot\left(\frac{w}{\sigma}\right)^{-5}$,

showing that again a power law distribution is obtained, however, with different differential densities for the two velocity components $w_{\|}, w_{\perp}$, i.e. not conformal with the type of isotropic power law distribution expected for quasiequilibrium states by Fisk and Gloeckler (2006). However, as these latter authors do emphasize in a more recent paper (Fisk and Gloeckler, 2007), in the framework of their model, no quasi-equilibrum state can be expected on the immediate downstream side of the shock, which would be required to establish clean power-law ion distributions.

In some way, Eq. (2) supports this fact, clearly demonstrating that upstream and downstream isotropy are not possible at a simple shock transition, and that additional physical mechanisms are definitely required to return to an isotropic distribution function on the downstream side. While this analysis is far from trivial, and will not be followed here, in the final section we will give some first ideas on what future results may be expected.

\section{The extent of the transition layer}

The question now may be arising within what time period $\tau_{m}$ the mirror-unstable anisotropic distribution function, resulting immediately after shock passage of the ions, may relaxate towards a marginally stable function which then may be close to the form expected by Fisk and Gloeckler (2006). For that purpose one simply has to study the growth rates of mirror-driven ion acoustic waves mainly propagating perpendicular to $\boldsymbol{B}$ (i.e. $k_{\perp} \gg k_{\|}$) due to the action of the mirror instability. These growth rates are given by (see Treumann and Baumjohann, 1997, pp. 55-60)

$\gamma_{m}=\sqrt{\frac{2}{\pi}} \frac{\beta_{\|}}{\beta_{\perp}^{2}}\left[\beta_{\perp}\left(\frac{\beta_{\perp}}{\beta_{\|}}-1\right)-1\right] k_{\|} c_{\|}$,

which may also be written as

$\gamma_{m}=\omega \sqrt{\frac{2}{\pi}}\left[\left(1-\frac{\beta_{\|}}{\beta_{\perp}}\right)-\frac{\beta_{\|}}{\beta_{\perp}^{2}}\right] \frac{c_{\|}}{v_{A}}$.
Here $c_{\|}$and $v_{A}$ denote the mean thermal speed of downstream ions parallel to $\boldsymbol{B}$ and the downstream Alfven velocity, respectively.

The fastest wave growth is found near the wave frequency $\omega=\omega_{2} i=e B_{2} / m_{i} c$. Thus one can find

$\gamma_{2 m}=\omega_{2 i} \sqrt{\frac{2}{\pi}} \frac{1}{A_{2}}\left[A_{2}-1-\frac{1}{\beta_{2 \perp}}\right] \frac{c_{2 \|}}{v_{2 A}}$.

Adopting $A_{2} \simeq 2.6$, we obtain

$\gamma_{2 m}=\omega_{2 i} \sqrt{\frac{1}{\pi}} \frac{1}{2.6}\left[1.6-\frac{1}{\beta_{2 \perp}}\right] \sqrt{\beta_{2 \perp}}$.

Evaluating this expression for a high $\beta$ - value downstream plasma, i.e. using $\beta_{2 \perp} \simeq 10$ one then arrives at

$\gamma_{2 m} \simeq \omega_{2 i} \sqrt{\frac{1}{\pi}} \frac{1.6}{2.6} \sqrt{\beta_{2 \perp}} \simeq \omega_{2 i}$.

The above relation states that the free mirror-mode energy may be coupled to the magnetosonic wave turbulence within about one ion gyroperiod. This can also be translated into a typical relaxation length $D_{m}$ the downstream plasma has to move while relaxation takes place. This length is given by:

$D_{m}=\tau_{2 m} U_{2}=\frac{c}{v_{A 2}} \frac{1}{\omega_{p i 2}} U_{2}=M_{a 2} \frac{c}{\omega_{p i 2}}$,

where $M_{a 2}$ and $\omega_{p i 2}$ denote the downstream Alfvenic Mach number and the downstream ion plasma frequency. Taking values $n_{2} \simeq 10^{-3} \mathrm{~cm}^{-3}$ and $M_{a 2} \simeq 0.3$ for the downstream plasma flow, we finally obtain

$D_{m}=M_{a 2} \frac{c}{\omega_{p i 2}}=0.3 \cdot 1.1 \cdot c \simeq 10^{5} \mathrm{~km} \simeq 10^{-3} \mathrm{AU}$

This means that ion relaxation from the mirror-mode unstable immediate downstream distribution function towards a marginally stable distribution may occur within a few thousandths of an AU downstream of the shock. Beyond a fraction of an $\mathrm{AU}$ the wind-convected plasma ions should be again in a nearly isotropic distribution similar to expectations of Fisk and Gloeckler (2006), although a non-negligible percentage of about 40 percent of the shock-generated entropy is now appearing in magneto-sonic turbulences. Similar results have also been derived by Liu et al. (2007), who have given arguments why mirror mode instabilities should operate downstream of the termination shock, and that some choices of upstream parameters, characterized by a high plasma- $\beta$ and a low Alfvenic Mach number $M_{a}$, result in a loss of entropy, and a "forbidden region" forms in parameter space. While they do not give quantitative estimates, they do point out that compressive MHD fluctuations should be generated in the heliosheath.

In fact, fluctuations of the compressive MHD-type appear to have been observed by Voyager-1, which, just a few years ago, crossed the solar wind termination shock and is currently taking data from the heliosheath, i.e. the downstream 
side of the termination shock. Burlaga et al. (2006a,b) have reported on chains of magnetic bumps and holes observed by the magnetometer on board of this spacecraft, which most probably could be identified as compressive MHD-wave turbulences, which had not been registered on the upstream side of the termination shock.

\section{Conclusions}

As we have shown, ions passing over the MHD termination shock initially attain a strongly pronounced pitchangle anisotropy. We have proven that this primary downstream distribution function is mirror-mode unstable with respect to driving magneto-acoustic waves. Up to 60 percent of the thermal ion energy, appearing just after shock passage in the mode perpendicular to the magnetic field, is converted further downstream by this instability into magnetoacoustic turbulence energy. These predictions could soon be confirmed after Voyager-2 passes over the termination shock, which is expected to happen any time now (Kiraly, 2005). There are two predicted sugnatures which could be identified, namely the emergence of anisotropic low energy ion distribution functions, and increased levels of compressive MHD fluctuations. We have estimated that the relaxation towards a new near-isotropic quasi-equilibrium distribution due to wave-particle coupling occurs within a downstream plasma passage of a tiny fraction of an AU. Only thereafter further isotropisation by pitchangle diffusion processes due to ion interactions with Alfvenic turbulences will drive the residual anisotropy of the quasi-equilibrium distribution towards a completely pitchangle isotropic one. The latter process will take place with typical pitchangle diffusion periods of the order of $\tau_{\mu \mu} \simeq 10^{8} \mathrm{sec}$ (see Chashei et al., 2005; Chashei and Fahr, 2005). This means that only after a downstream passage of the solar wind plasma over a distance of $L_{\mu \mu} \simeq \tau_{\mu \mu} U_{2} \simeq 60 \mathrm{AU}$ the plasma ions finally will have reached a fully pitchangle isotropic distribution. Nevertheless nearly immediately after shock passage a relevant fraction of the shock-entropized energy reappears in the form of magneto-acoustic turbulences, meaning the level of magneto-acoustic wave power is much higher than expected up to now. This prediction appears to be nicely confirmed by Voyager-1 magnetometer results, which show nonlinear compressive MHD fluctuations (Burlaga et al., 2006a,b).

Up to now it was tacitly assumed in the literature that Alfvenic and magnetosonic turbulences are simply convected over the shock as already described in a theory by McKenzie and Westphal (1969). For moderate MHD shocks, this then leads to the result that the upstream normalized magnetosonic turbulence power should be reduced by a factor 0.1 on the downstream side, whereas the Alfvenic turbulence power is more or less conserved (Chashei et al., 2005). This would mean that ion energy diffusion by interaction with large scale magnetosonic waves downstream of the shock, in view of a formula given for the velocity diffusion coefficient $D_{w w}$ (see Toptygin, 1983, pg. 199) should be reduced. This formula has been applied to the downstream plasma region by Chalov et al. (2003) and leads to

$D_{2, w w} \sim \frac{\sqrt{\left\langle\delta U_{2}^{2}\right\rangle}}{L_{2, m}} \sim \frac{0.1 \sqrt{\left\langle\delta U_{1}^{2}\right\rangle}}{L_{2, m}} \sim 0.1 \frac{L_{1, m}}{L_{2, m}} D_{1, w w}$,

where $\left\langle\delta U_{1}^{2}\right\rangle$ and $L_{m}$ denote the large-scale correlation average of magnetosonic fluctuations and the correlation length of these fluctuations, respectively. For the latter quantity, one finds

$L_{m} \geq \frac{w \lambda \|}{3 U}$,

with $\lambda_{\|} \sim 3 D_{A} / w \sim\left\langle\delta B_{A}^{2}\right\rangle / w$ being the mean free path with respect to scattering by short wavelength Alfven fluctuations and $D_{A}$ being the velocity diffusion coefficient due to ion interactions with Alfvenic fluctuations. Thus one obtains

$D_{2, w w} \sim 0.1 \frac{\left\langle\delta B_{A}^{2}\right\rangle_{1} U_{2}}{\left\langle\delta B_{A}^{2}\right\rangle_{2} U_{1}} D_{1, w w} \simeq \frac{0.1}{\sigma} D_{1, w w}$

This means that, if only turbulence convection over the shock would occur, the effect of velocity diffusion or transit time damping (see Fisk, 1976; Schwadron et al., 1996; Fisk and Gloeckler, 2006) would appear to be strongly reduced downstream of the shock. In view, however, of the turbulence power generation due to mirror-mode unstable ion distribution functions which we have described, this figure may be changing to the opposite, because under these latter conditions the magnetoacoustic turbulence levels might be increased to $\left\langle\delta U_{2}^{2}\right\rangle \simeq \Gamma_{m}\left\langle U_{1}-U_{2}\right\rangle^{2}$, leading to

$$
\begin{aligned}
D_{2, w w} & \sim \frac{\Gamma_{m}\left\langle U_{1}-U_{2}\right\rangle^{2}}{\left\langle\delta U_{1}^{2}\right\rangle} D_{1, w w} \\
& =\frac{\Gamma_{m}\left\langle U_{1}-U_{2}\right\rangle^{2}}{U_{1}^{2}} \frac{U_{1}^{2}}{\left\langle\delta U_{1}^{2}\right\rangle} D_{1, w w}
\end{aligned}
$$

where the factor $\Gamma_{m}$ denotes the fraction of energy reappearing in magnetosonic fluctuation power. This factor can be estimated by the fraction $\Gamma_{k i n} \simeq U_{2}^{2} /\left(U_{1}^{2}-U_{2}^{2}\right)$ of dissipated energy appearing in the form of kinetic energy and then is given by

$\Gamma_{m} \simeq 0.4 \cdot \Gamma_{\perp}=0.4 \frac{1-\Gamma_{k i n}}{A_{2}+1}=0.4 \frac{1-\frac{1}{\sigma^{2}-1}}{\sigma+1}$

Calculating $\Gamma_{m}$ from this equation, and adopting a compression ratio of $\sigma \simeq 3$, we then obtain $\Gamma_{m} \simeq 0.09$.

In their paper, Chalov et al. (2003) have used the following representation for upstream magnetosonic fluctuations

$\frac{\left\langle\delta U_{1}^{2}\right\rangle}{U_{1}^{2}}=0.25 \cdot\left(\frac{r_{T S}}{r_{E}}\right)^{-1.4} \simeq 10^{-3}$ 
where $r_{T S}=90 r_{E}$ and $r_{E}$ are the solar distances of the termination shock and of the Earth, respectively. With the above assumptions, we finally arrive at

$$
\begin{aligned}
D_{2, w w} & \sim \frac{\Gamma_{m}\left\langle U_{1}-U_{2}\right\rangle^{2}}{U_{1}^{2}} \frac{U_{1}^{2}}{\left\langle\delta U_{1}^{2}\right\rangle} D_{1, w w} \\
& =0.09 \cdot\left(1-\frac{2}{\sigma}+\frac{1}{\sigma^{2}}\right) 10^{3} D_{1, w w} \simeq 70 D_{1, w w}
\end{aligned}
$$

In their study of the pick-up ion transport in the heliosheath, Chalov et al. (1997) have shown how such an enhancement of the velocity diffusion coefficient acts via the phasespace transport equation on the ion distribution function, while these ions are convected downstream with the solar wind plasma flow. As they can show a strong enhancement of suprathermal tails can be clearly seen as a reaction to that.

Finally it is interesting to notice that this enhancement of magnetosonic fluctuation power also has interesting consequences for the acceleration times of anomalous cosmic rays (ACR's). Characteristic ACR acceleration times have been derived by Drury (1983) in the form

$\tau_{A C R}=\frac{3}{U_{1}-U_{2}}\left(\frac{\kappa_{1}}{U_{1}}+\frac{\kappa_{2}}{U_{2}}\right)$,

where $\kappa_{1,2}$ are the spatial diffusion coefficients of ACR particles. The above formula has been applied by Scherer et al. (1998) to the problem of ACR acceleration at the termination shock with the assumption that $\sigma=\kappa_{1} / \kappa_{2}$ can be adopted and then delivers acceleration times

$\tau_{A C R}^{0}=\frac{6 \sigma}{\sigma-1}\left(\frac{\kappa_{1}}{U_{1}^{2}}\right)$

of about or larger than a year to arrive at ACR energies of $70 \mathrm{MeV}$ or higher.

Under the aspects of turbulence generation downstream of the shock, however, this result needs to be substantially revised yielding

$\tau_{A C R}=\frac{3}{U_{1}-U_{2}} \frac{\kappa_{1}}{U_{1}}\left(1+\sigma \frac{\lambda_{\| 2}}{\lambda_{\| 1}}\right) \simeq \frac{1}{2} \tau_{A C R}^{0}$

meaning that ACR acceleration time periods might show to be only half of what has been calculated by Scherer et al. (1998).

Acknowledgements. We very much appreciate financial support by the Deutsche Forschungsgemeinschaft (DFG) granted to us in the frame of the project Fa-97/31-1.

Edited by: H. Fichtner

Reviewed by: L. Fisk and another referee

\section{References}

Baumjohann, W. and Treumann, R. A.: Basic space plasma physics, London: Imperial College Press, 1996.
Burlaga, L. F., Ness, N. F., and Acuna, M. H.: Magnetic Fields in the Heliosheath: Voyager 1 Observations, Astrophys. J., 642, 584-592, 2006a.

Burlaga, L. F., Ness, N. F., and Acuna, M. H.: Trains of magnetic holes and magnetic humps in the heliosheath, Geophys. Res. Lett., 33, L21 106, doi:10.1029/2006GL027276, 2006 b.

Chalov, S. V., Fahr, H.-J., and Izmodeov, V.: Spectra of energized pick-up ions upstream of the two-dimensional heliospheric termination shock. II. Acceleration by Alfvenic and by large-scale solar wind turbulences, å, 320, 659-671, 1997.

Chalov, S. V., Fahr, H.-J., and Izmodeov, V.: Evolution of pickup ion spectra in the inner heliosheath and their diagnostics by energetic neutral atom fluxes, J. Geophys. Res., 108(6), 1266$1274,2003$.

Chashei, I. V. and Fahr, H.-J.: Ion relaxation processes in the heliospheric interface: how perturbed are ion distribution functions?, Adv. Sp. Res., 35, 2078-2083, 2005.

Chashei, I. V., Fahr, H.-J., and Lay, G.: Non-Equilibrium Distribution Functions in the Heliospheric Interface and Their Relaxation by Local Wave Particle Interactions, Solar Physics, 226, 163-185, 2005.

Diver, D. A.: A Plasma Formulary for Physics, Technology and Astrophysics, Hoboken, New Jersey: John Wiley, 2001.

Drury, L. O. C.: An introduction to the theory of diffusive acceleration of energetic particles in tenuous plasmas, Rep. Prog. Phys, 46, 973-1016, 1983.

Erkaev, N. V., Vogl, D. F., and Biernat, H. K.: Solution for jump conditions at fast shocks in an anisotropic plasma, J. Plasma Physics, 64, 561-578, 2000.

Fahr, H.-J. and Lay, G.: Remote diagnostic of the heliospheric termination shock using neutralized post shock pick-up ions as messengers, A\&A, 356, 327-334, 2000.

Fahr, H.-J. and Siewert, M.: Kinetic study of the ion passage over the solar wind termination shock, A\&A, 458, 13-20, doi: 10.1051/0004-6361:20065540, 2006.

Fisk, L. A.: The acceleration of energetic particles in the interplanetary medium by transit-time damping, J. Geophys. Res., 81, 4633, 1976.

Fisk, L. A. and Gloeckler, G.: The common spectrum for accelerated ions in the quiet-time solar wind, Astrophys. J., 640, L79L82, 2006.

Fisk, L. A. and Gloeckler, G.: Thermodynamic constraints on stochastic acceleration in compressional turbulence, PNAS, 104, 5749-5754, doi:10.1073/pnas.0700881104, 2007.

Gombosi, T. I.: Physics of the space Environment, New York: Cambridge Univ. Press, 1998.

Hudson, P. D.: Discontinuities in an anisotropic plasma and their identification in the solar wind, Plan. Sp. Sc., 18, 1611-1622, 1970.

Kiraly, P.: The way out of the bubble: Implications of recent Voyager-1 data, in: Proc. 29th Intern. Cosmic Ray Conf., 1, 339342, 2005.

Krasnoselskikh, V. V., Lemberge, B., Savoni, P., and Lobzin, V. V.: Nonstationarity of strong collisionless quasiperpendicular shocks: Theory and full particle numerical simulations, Phys. Plasmas, 9, 1192-1209, 2002.

Lee, L. C., Wu, C. S., and Hu, X. W.: Increase of ion kinetic temperature across a collisionless shock: I. A new mechanism, Geophys. Res. Lett., 13, 209-213, 1986. 
Lembege, B., Giacalone, J., Scholer, M., Hada, T., Hoshino, M., Krasnoselskikh, V., Kucharek, H., Savoini, P., and Terasawa, T.: Selected problems of collisionless shock physics, Sp. Sc. Rev., 110, 161-226, 2004.

Liu, Y., Richardson, J. D., Belcher, J. W., and Kasper, J. C.: Temperature anisotropy in a shocked plasma: Mirror-mode instabilities in the heliosheath, Astrophys. J., 659, L65-L68, 2007.

Lobzin, V. V., Krasnoselskikh, V. V., Bosquel, J.-M., Princon, J. L., Schwartz, S. J., and Dunlop, M.: Nonstationarity and reformation of high-Mach-number quasiperpendicular shocks: Cluster observations, Geophys. Res. Lett., 34, L05 107-L05 113, 2007.

McKenzie, J. F. and Westphal, K. O.: Transmission of Alfven waves through the Earth's bow shock, Plan. Sp. Sc., 17, 1029-1037, 1969.

Quest, K. B.: Simulations of High Mach Number Perpendicular Shock With Resistive Electrons, J. Geophys. Res., 91, 88058815, 1986.

Scherer, K., Fichtner, H., and Fahr, H.-J.: The acceleration time of anomalous cosmic rays - Observational constraints from Pioneer 10 data, J. Geophys. Res., 103, 2105-2113, 1998.

Scholer, M.: Kinetic structure of the heliospheric termination shock and implications for pickup ion injection, in: Physics of the outer heliosphere: 3rd Int. IGPP Conf., CP719 of AIP Conf. Proceedings, 311-316, 2004.

Scholer, M. and Matsukiyo, S.: Nonstationarity of quasi-perpendicular shocks: a comparision of full particle simulations with different ion to electron mass ratio, Ann. Geophys., 22, 23452351, 2004.
Scholer, M., Shinohara, I., and Matsukiyo, S.: Quasi-perpendicular shocks: Length scale of the cross-shock potential, shock reformation, and implication for shock surfing, J. Geophys. Res., 108, 1014-1022, 2003.

Schwadron, N. A., Fisk, L. A., and Gloeckler, G.: Statistical acceleration of interstellar pick-up ions in co-rotating interaction regions, Geophys. Res. Lett., 23, 2871-2875, 1996.

Siewert, M. and Fahr, H.-J.: Analytic distribution functions for an ion plasma crossing an MHD shock, A\&A, 463, 799-805, doi: 10.1051/0004-6361:20066233, 2007a.

Siewert, M. and Fahr, H.-J.: Full Boltzmann-kinetical treatment of an ion plasma crossing an MHD shock: parallel and non-parallel cases, A\&A, 471, 7-15, doi:10.1051/0004-6361: 20077413, 2007b.

Toptygin, I. N.: Cosmic rays in interplanetary magnetic fields, Norwell/Mass.: D. Reidel Publ., 1983.

Treumann, R. A. and Baumjohann, W.: Advanced space plasma physics, London: Imp. College Press, 1997.

Zank, G. P., Ao, X., Axford, W. I., Florinski, V., Li, G., and le Roux, J. A.: Structure and properties of the termination shock, in: Physics of the outer heliosphere: 3rd Int. IGPP Conf., CP719 of AIP Conf. Proceedings, 329-341, 2004. 